\title{
Two-Dimensional Micro-Hartmann Gas Flows
}

\author{
Chunpei Cai and Khaleel R.A. Khasawneh \\ Department of Mechanical \& Aerospace Engineering \\ New Mexico State University, Las Cruces, New Mexico, 88003-8001, U.S.A. \\ ccai@nmsu.edu
}

\begin{abstract}
We analyze and simulate a near continuum MagnetoGasDynamic(MGD) flow inside a two-dimensional microchannel with a low magnetic Reynolds number assumption. Complex physics such as rarefication, electric and magnetic effects are considered in the asymptotic solutions. This work represents an extension from the classical Hartmann flow in a two-dimensional channel of infinite length to a microchannel of finite length. We obtain a non-dimensional equation that relates the pressure ratio, Reynolds number, Mach number, magnetic Reynolds number and magnetic force number. We also solved for asymptotic solutions of compressible gas flow based on the velocity-slip and temperature-jump wall boundary conditions while maintaining a consistent quasi-isothermal assumption. Numerical solutions of the same formulation are obtained for validation of the present analytical solutions.
\end{abstract}

Keywords: multi-scales, micro-flows, rarefication flows, Hartmann flows.

\section{Introduction}

Microchannels are important components for many Micro-Electro-Mechanical Systems(MEMS).The study of gaseous flows inside microchannels has been an interesting research topic. There are many reports in the literature about gas flows in microchannels and micro-tubes. [1] A microchannel for fuel cells, for example, is of dimensions of micrometers, whereas a channel of conventional dimensions is of order of centimeters to meters. It is well accepted that for near continuum gas flows through microchannels, the Navier-Stokes equations are valid if a slip wall boundary condition is used. Many researchers have obtained theoretical solutions for the flow distributions along a microchannel with an isothermal assumption, including the pioneering work by Arkilic et al, [1] and Zohar et al. [2 Numerically, there are many methods for simulating compressible flows in a microchannel: with the direct simulation Monte Carlo method, the Information Preservation method, 3] gas-kinetic BGK-Burnett equation solutions. 4] Discussions of thermal heating effects are reported as well. [5]

One important problem related to gas flow inside a microchannel is conductive gas flows under external magnetic and electric fields, or MagnetoGasDynamic(MGD) flows. These fields can significantly affect the internal gas flow field, while different placements of electric field enables the channel to perform

G. Allen et al. (Eds.): ICCS 2009, Part I, LNCS 5544, pp. 655-664, 2009.

(C) Springer-Verlag Berlin Heidelberg 2009 
as a generator, a pump, a flux meter, or an accelerator. [6] This work is an extension from the classical Hartmann flow 6 , to a flow in a microchannel of finite length with the follow differences. First, for pressure driven gas flows inside a microchannel, usually there are large density changes inside the microchannel, and the average velocity can not remain unchanged in order to maintain a constant mass flow rate. Second, the boundary conditions are different. For Hartmann flow, there is essentially no variation in the flow direction; wall boundary conditions are nonslip and constant temperature; one can use periodic boundary conditions (except for pressure) at the inlet and outlet. 7] However, for microchannel flows with density variations and rarefication effects, we will not use periodic boundary conditions at inlet and outlet; further, we will specify a set of general velocity-slip and temperature-jump wall boundary conditions, which include the continuum no-slip and temperature boundary conditions as a special case. Third, the pressure gradient inside a microchannel of finite length is not assumed constant throughout. Simply taking a linear pressure distribution assumption (after the Hartmann flow) for the present microchannel flow will result in an inaccurate solution.

The work in this paper is a natural extension from two previous work by Arkilic et al [1] and Cai et al. [5] Along the same vein, the present study follows Cai's previous approach [5] for treatments of microchannel MGD flows. A few works appeared in recent years in the same area of MGD microchannel flow. 89. There are some differences between the present approach and others: 1. Our study includes a governing equation for temperature necessitated by the Joule heating effects to obtain the temperature field; [5] by comparison, the past work completely neglects the energy equation. 2. We utilize the low magnetic Reynolds number assumption, which is crucial to simplify the MGD equations; we have not noticed any previous treatment for the same problem in the literature. Without this assumption, the magnetic field variations in general should be considered; thus it is unlikely that such asymptotic approach can be consistently formulated. 3. According to the X-momentum equation, we provided the governing relation between the Re and Ma numbers and two other nondimensional parameters for magnetic and electric fields. We then conducted a consistent order of magnitude analysis. 4. We obtain a full set of asymptotic solutions in a microchannel flow. The nonlinear pressure solution implies that without it the solution for $\mathrm{U}$-Velocity is incorrect.

\section{Problem Description and Order Estimations}

As illustrated by Figure 1, a microchannel has a height of $2 d$ and a length of $L$, and the average compressible gas properties of pressure, density, velocity and number density are $p_{o}, \rho_{o}, U_{o}, n_{o}$ at the channel outlet. The averaged outlet quantities are adopted to normalize the following governing equations and boundary conditions. The inlet pressure is several times larger than the outlet pressure, their pressure ratio is denoted as $P$. The coordinate origin is set at the inlet 
center point, and the $\mathrm{X}$-axis is along the channel centerline, the $\mathrm{Y}$-axis is along the direction normal to the channel wall. Further we assume:

1. External magnetic field is fixed at a value $\left(0, B_{0}, 0\right)$, along the Y-direction only, electric field is along the $\mathrm{Z}$ direction only $\left(0,0, E_{z}\right)$.

2. The flow is well approximated with a quasi-isothermal assumption. [5] As such, gas viscosity, $\mu$, thermal conductivity, $k$, magnetic permeability, $\mu_{m}$, and electric conductivity, $\sigma$, are treated as constants.

3. The magnetic Reynolds number, $R_{\sigma}=(2 d) U_{o} \mu_{o} \sigma$, is very small. It renders a negligible induced magnetic field, $B_{x}$ compared with $B_{0}$.

4. The flow is two-dimensional, hence $\partial / \partial z=0, w=0$.

5. We assume the electric field is linked with the magnetic field with $E_{z}=$ $-K u_{o} B_{0}$, and $0<K<1$. [7] Hence, we can combine the electric field into the magnetic field to estimate orders of magnitude for different forces.

6. The channel is not short, which means $\epsilon=2 d / L$ is small.

A proper order estimation for several nondimensional parameters is crucial to simplify the governing equations. Hence, we need first to estimate the orders of magnitude for several nondimensional parameters, including Mach number, $M a=U_{o} / \sqrt{\gamma R T_{o}}$, Reynolds number, $R e=(2 d) \rho_{o} U_{o} / \mu$, magnetic force number, $R_{b}=B_{0}^{2} /\left(\rho_{o} U_{o}^{2} \mu_{m}\right)$, magnetic Reynolds number, $R e_{\sigma}=(2 d) U_{o} \mu_{o} \sigma_{o}$, Knudsen number, $K n=\lambda /(2 d) \sim \sqrt{\frac{\pi \gamma}{2}} \frac{M a}{R e}$, and Hartmann number, $H_{a}=\sqrt{R e R_{\sigma} R_{b}}$. These nondimensional numbers are based on the quantities at the outlet, and the external magnetic field.

By choosing the whole flow domain as an integral domain [5] and exercising the X-momentum equation globally, we obtain:

$$
2 d\left(P_{o}-P_{i}+\rho_{o} U_{o}^{2}-\rho_{i} U_{i}^{2}\right)=\mu \frac{U_{i}+U_{o}}{2 d} 2 L-\sigma B_{0}\left(E_{z}+\frac{U_{o}+U_{i}}{2} B_{0}\right)(2 d) L
$$

Further, we drop those terms with $U_{i}$ because $U_{i}<<U_{o}$ for a pressure driven gas flow inside a microchannel. Then, the following simple relation is obtained:

$$
\epsilon\left(1+1 /\left(\gamma M a^{2}\right)(1-1 / P)\right) \sim 1 / R e+R_{\sigma} R_{b}
$$

where $P=p_{i} / p_{o}$, and $\epsilon=2 d / L$. The parameter $K$, which is a key physical factor for the microchannel flow, presents the ratio between the electric field and the magnetic field. Here it is considered to be the same order, or less than, the magnetic field effects, and it is combined into the factor $R_{\sigma} R_{b}$.

Eqn.(1) contains four nontrivial terms: 1) the momentum change term, represented by $\epsilon$ on the left hand side; 2) the pressure drop term applying at the channel inlet and outlet, $\epsilon /\left(\gamma M_{a}^{2}\right)(1-1 / P)$, on the left hand side; 3$)$ the viscous force along the wall surfaces, $1 / R e$, on the right hand side; and 4) the magnetic/electric force which applies to the whole domain, $R_{\sigma} R_{b}$, on the right hand side. There are many choices to balance these terms: all terms can share the same order of magnitudes; or three terms share the larger order while the other term is smaller; or two of the four terms are larger while the other two terms are equally smaller; or two of the four terms are large, one term is relatively small and the 
other term is the smallest. Hence, from the above four permutations, there will be at least $1+C_{4}^{3}+C_{4}^{2} C_{2}^{2}+C_{4}^{2} C_{2}^{1}=23$ classes of combinations, and most of these combinations can have many detailed subclasses as well. Here we are interested in investigating the interactions among viscous stress, pressure drop and magnetic/electric field effects. Different parameter orders may result in different simplified governing equations and different flow solutions, including hypersonic, supersonic and transonic flows, as we illustrated in our previous paper. 5] Here we are specially interested in slow MGD flows under effects of strong pressure difference, strong viscous effects along channel wall, and strong MGD force. In this study, we select two viable cases with the following parameter combinations which satisfy (1):

1. $R e \sim \epsilon, M_{a} \sim \epsilon, K n \sim 1 ; R_{b} \sim 1 / \epsilon^{2}, R_{\sigma} \sim \epsilon ; H_{a} \sim 1$;

2. $R e \sim 1, M_{a} \sim \epsilon^{1 / 2}, K n \sim \epsilon^{1 / 2} ; R_{b} \sim 1 / \epsilon, R_{\sigma} \sim \epsilon ; H_{a} \sim 1$;

Previously [5] we showed that the Reynolds and Mach numbers in these two cases render a balance between the viscous effects and the pressure drop term. We intend to set $R_{\sigma}$ at least one order smaller than $R_{b}$ to create a very small induced magnetic field, but the magnetic interaction factor $Q=R_{\sigma} R_{b}$ is assumed to be as strong as the terms for viscous force and pressure drop.

\section{$3 \quad$ Asymptotic Solutions}

Here the flow quantities are normalized with the averaged properties at the outlet, and the $\mathrm{X}-, \mathrm{Y}$-coordinates are normalized with $L, 2 d$ correspondingly. 1.5. With the two sets of parameters previously selected, the non-dimensional normalized governing equations and boundary conditions are: 10]

$$
\begin{gathered}
\epsilon \frac{\partial(\rho u)}{\partial x}+\frac{\partial(\rho v)}{\partial y}=0, p=\rho T=n T \\
\epsilon \frac{\partial}{\partial x}\left(\rho u u+\frac{p}{\gamma M_{a}^{2}}\right)+\frac{\partial(\rho v u)}{\partial y}=\frac{1}{R e}\left(\epsilon^{2} \frac{4}{3} \frac{\partial^{2} u}{\partial x^{2}}+\frac{\partial^{2} u}{\partial y^{2}}+\frac{1}{3} \epsilon \frac{\partial^{2} v}{\partial x \partial y}\right)+Q(K-u) \\
\epsilon \frac{\partial(\rho u v)}{\partial x}+\frac{\partial}{\partial y}\left(\rho v^{2}+\frac{p}{\gamma M_{a}^{2}}\right)=\frac{1}{R e}\left(\epsilon^{2} \frac{\partial^{2} v}{\partial x^{2}}+\frac{4}{3} \frac{\partial^{2} v}{\partial y^{2}}+\epsilon \frac{\partial^{2} u}{\partial x \partial y}\right) \\
\epsilon \rho u \frac{\partial T}{\partial x}+\rho v \frac{\partial T}{\partial y}=\epsilon \frac{\gamma-1}{\gamma} u \frac{\partial p}{\partial x}+\frac{\gamma-1}{\gamma} v \frac{\partial p}{\partial y}+\frac{1}{R e P r}\left(\epsilon^{2} \frac{\partial^{2} T}{\partial x^{2}}+\frac{\partial^{2} T}{\partial y^{2}}\right)+\frac{(\gamma-1) M^{2}}{R e}\left[2\left(\frac{\partial u}{\partial x}\right)^{2} \epsilon^{2}\right. \\
\left.+2\left(\frac{\partial v}{\partial y}\right)^{2}+\left(\epsilon \frac{\partial v}{\partial x}+\frac{\partial u}{\partial y}\right)^{2}-\frac{2}{3}\left(\frac{\partial u}{\partial x} \epsilon+\frac{\partial v}{\partial y}\right)^{2}\right]+Q K(K-u) \\
\left.u_{1}(x, y)\right|_{y= \pm 1 / 2}=\left.\left.\Theta_{u} K n(x)\right|_{y= \pm 1 / 2} \frac{\partial u_{1}(x, y)}{\partial n}\right|_{y= \pm 1 / 2} \\
\left.T\right|_{y= \pm 1 / 2}-T_{w}=\Theta_{T} \frac{2 \gamma}{\operatorname{Pr}(\gamma+1)} K n(x)\left(\frac{\partial T}{\partial n}\right)_{y= \pm 1 / 2}
\end{gathered}
$$


where the relations $|v|<|u|$ and $\left|B_{x}\right|<<\left|B_{0}\right|$ are used to simplify the source terms in the momentum and energy equations, $\Theta_{u}=\left(2-\sigma_{u}\right) / \sigma_{u}$ and $\Theta_{T}=$ $\left(2-\sigma_{T}\right) / \sigma_{T}$. In (6) for the nondimensional velocity boundary condition, a temperature gradient term is omitted because it is smaller than the velocity gradient with the two specific groups of nondimensional parameters.

Further, we assume the following expansions $u=u_{1}+\epsilon u_{2}+\ldots, v=v_{1}+\epsilon v_{2}+$ $\ldots, p=p_{1}+\epsilon p_{2}+\ldots, \rho=\rho_{1}+\epsilon \rho_{2}+\ldots, n=n_{1}+\epsilon n_{2}+\ldots, T=1+\epsilon T_{2}$. The last one implies a quasi-isothermal assumption. 55 Specifically, from $p(x, y)=$ $\rho(x, y)\left(1+\epsilon T_{2}(x, y)\right)$, we obtain $p_{1}=\rho_{1}$ as the zero order relation and the isothermal assumption proposed by Arkilic et al[1] is relaxed.

For the two sets of parameters, the Y-momentum equation simplifies as:

$$
\frac{\partial p_{1}(x, y)}{\partial y}=0
$$

With the low magnetic Reynolds number assumption, the magnetic term is at least one order smaller than the pressure term. From (8) we have $p_{1}(x, y)=$ $p_{1}(x)$, which greatly simplifies the following derivations.

The simplified X-momentum equation is: 6 ]

$$
\frac{\partial^{2} u_{1}}{\partial y^{2}}-H_{a}^{2} u_{1}=-H_{a}^{2} K+\frac{\epsilon R e}{\gamma M_{a}^{2}} \frac{d p_{1}}{d x}
$$

The slip wall boundary conditions result in different solutions from the classical Hartmann flow solutions. The final U-velocity solution is,

$$
u_{1}(x, y)=C_{1} \frac{\epsilon R e}{\gamma M^{2}} \frac{d p_{1}}{d x} \cosh \left(H_{a} y\right)-\frac{\epsilon R e}{H_{a}^{2} \gamma M^{2}} \frac{d p_{1}}{d x}+K
$$

where $C_{1}=\frac{1}{H_{a}^{2} C_{2}}-\frac{K \gamma M^{2}}{C_{2} \epsilon \operatorname{Re}\left(d p_{1} / d x\right)}, C_{2}=\cosh \left(\frac{H_{a}}{2}\right)+\Theta_{u} K n(x) \sinh \left(\frac{H_{a}}{2}\right) H_{a}$.

The zero-order solution for the $\mathrm{V}$-velocity is obtained from the zero-order of the continuity equation, $v_{1}=0$, and the next order can be obtained from the continuity equation, by using the relation $\rho_{1}=p_{1}$ :

$$
\frac{\partial\left(p_{1}(x) u_{1}(x, y)\right)}{\partial x}+\frac{\partial\left(p_{1}(x) v_{2}(x, y)\right)}{\partial y}=0
$$

and the result is:

$$
\begin{aligned}
v_{2}(x, y)= & A_{1} \sinh \left(H_{a} y\right)\left[-\frac{1}{2} \frac{d^{2} p_{1}^{2}}{p_{1} d x^{2}}+A_{2} \frac{d^{2} p_{1}}{p_{1} d x^{2}}\right]+A_{3} K \frac{d p_{1}}{p_{1} d x} \sinh \left(H_{a} y\right) \\
& +y \frac{\epsilon R e}{2 H_{a}^{2} \gamma M^{2}} \frac{d^{2} p^{2}}{p_{1} d x^{2}}-\frac{d p_{1}}{p_{1} d x} K y
\end{aligned}
$$

where $A_{1}=\frac{\epsilon R e}{\gamma M^{2} H_{a}^{3} \cosh \left(H_{a} / 2\right)}, A_{2}=\Theta_{u} H_{a} K n_{o} \tanh \left(H_{a} / 2\right), A_{3}=\frac{1}{H_{a} \cosh \left(H_{a} / 2\right)}$. The temperature distribution can be obtained by the following simplified energy equation and the temperature-jump wall boundary conditions:

$$
\frac{1}{R e P r} \frac{\partial^{2} T_{2}}{\partial y^{2}}=-\frac{\gamma-1}{\gamma} u_{1} \frac{d p_{1}}{d x}-\frac{(\gamma-1) M^{2}}{\epsilon R e}\left(\frac{\partial u_{1}(x, y)}{\partial y}\right)^{2}-Q K(K-U)
$$


The solution for the temperature field is:

$$
T_{2}(x, y)=\operatorname{Re} \operatorname{Pr}\left(\frac{N_{3}}{H_{a}^{2}} \cosh \left(H_{a} y\right)+\frac{N_{5}}{4 H_{a}^{2}} \cosh \left(2 H_{a} y\right)+\frac{N_{6}}{2} y^{2}+N_{8}\right)
$$

where $N_{8}$ is determined by the temperature jump boundary condition:

$$
\begin{aligned}
N_{8}= & \frac{T_{w}-1}{\epsilon R e P r}-\frac{N_{3}}{H_{2}^{2}} \cosh \left(\frac{H_{a}}{2}\right)-\frac{N_{5}}{4 H_{a}^{2}} \cosh \left(H_{a}\right)-\frac{N_{6}}{8}-\Theta_{T} \frac{2 \gamma K n(x)}{\operatorname{Pr}(\gamma+1)} \\
& {\left[\frac{N_{3}}{H_{a}} \sinh \left(\frac{H_{a}}{2}\right)+\frac{N_{5}}{2 H_{a}} \sinh \left(H_{a}\right)+\frac{N_{6}}{2}\right] }
\end{aligned}
$$

Moreover $N_{1}=C_{1} \frac{\epsilon R e}{\gamma M^{2}} \frac{d p_{1}}{d x}, N_{2}=-\frac{\epsilon R e}{H_{a}^{2} \gamma M^{2}} \frac{d p_{1}}{d x}+K, N_{3}=-\frac{\gamma-1}{\gamma} N_{1} \frac{d p_{1}}{d x}+Q K N_{1}$, $N_{4}=-\frac{\gamma-1}{\gamma} N_{2} \frac{d p_{1}}{d x}-Q K^{2}+Q K N_{2}, N_{5}=-\frac{(\gamma-1) M^{2}}{2 \epsilon R e} N_{1}^{2} H_{a}^{2}, N_{6}=N_{4}-N_{5}$. The density distribution is approximated as $\rho(x, y)=p_{1}(x) /\left(1+\epsilon T_{2}(x, y)\right.$. It is evident that the density is not constant at any specific station with $\mathrm{x}=$ constant.

The pressure distribution is crucial to the whole set of solution since $p(x)$ and its gradients dominate the coefficients for $u(x, y), v(x, y)$ and $T(x, y)$. Evaluating the $\mathrm{V}$-velocity along the upper channel wall results in the following equation:

$$
D_{1} \frac{d^{2} p_{1}^{2}}{d x^{2}}+D_{2} \frac{d^{2} p_{1}}{d x^{2}}+D_{3} K \frac{d p_{1}}{d x}=0
$$

where $D_{1}=-\left(\frac{\tanh \left(H_{a} / 2\right)}{2 H_{a}^{3}}-\frac{1}{4 H_{a}^{2}}\right) \frac{\epsilon R e}{\gamma M^{2}}, D_{2}=\frac{\tanh ^{2}\left(H_{a} / 2\right)}{H_{a}^{2}} \frac{\epsilon R e}{\gamma M^{2}} \Theta_{u} K n_{o}$ and $D_{3}=$ $-\frac{1}{2}+\frac{\tanh \left(H_{a} / 2\right)}{H_{a}}$. The boundary conditions, $p_{1}(0)=P$ and $p_{1}(1)=1$ lead to the following exact solutions to the nonlinear ordinary differential equation:

1. if $K=0$, i.e., in the absence of the electric field effect:

$$
p_{1}(x)=\frac{-D_{2}+\sqrt{D_{2}^{2}+4 D_{1}\left(D_{1} P^{2}+D_{2} P+D_{4} x\right)}}{2 D_{1}}
$$

where $D_{4}=D_{1}\left(1-P^{2}\right)+D_{2}(1-P)$.

2. if $K>0$, i.e., with a uniform electric field:

$$
2 D_{1} p_{1}(x)+\left(\frac{2 D_{1} G_{1}}{D_{3} K}+D_{2}\right) \ln \left[D_{3} K p_{1}(x)-G_{1}\right]=-x D_{3} K+G_{2} D_{3} K
$$

The two boundary conditions exactly determine the coefficients $G_{1}$ and $G_{2}$ in the above solution: $G_{2}=\frac{2 D_{1}}{D_{3} K}+\left(\frac{2 D_{1} G_{1}}{D_{3}^{2} K^{2}}+\frac{D_{2}}{D_{3} K}\right) \ln \left(D_{3} K-G_{1}\right)+1$ and $2 D_{1}(P-1)-D_{3} K=\left(\frac{2 D_{1} G_{1}}{D_{3} K}+D_{2}\right) \ln \left(\frac{D_{3} K-G_{1}}{D_{3} K P-G_{1}}\right)$. Solving for $G_{1}$ requires an iterative method. However, for a given set of non-dimensional numbers, $G_{1}$ and $G_{2}$ are completely determined.

\section{Numerical Validations}

We perform numerical computations to solve the low magnetic Reynolds number MGD equations, and compare the results with the corresponding analytical results. The computations are performed with a well-tested general Navier-Stokes 
equation solver. The flow parameters are: $\epsilon=0.06, L=20 \mu \mathrm{m}, p_{1}=2 \mathrm{~atm}$, $p_{2}=1 \mathrm{~atm}, T_{1}=300 \mathrm{~K}, \sigma_{u}=\sigma_{T}=0.85$, oxygen gas, $\gamma=1.4, \operatorname{Pr}=0.72$, $\mu=1.919 \times 10^{-5}$ sec. $N / m^{2}$, wall temperature $T_{w}=300 K, R_{b}=1 / \epsilon$ and $R_{\sigma}=\epsilon$. When the magnetic field is enabled, $K$ is chosen to be $0.0,0.5$ or 0.9 . Many of these simulation parameters are the same as those in our previous paper [5] on a neutral gas flow case.

Figures 2-3 correspond to a case with $K=0.5$, the so-called "impedance match" case for Hartmann flow. 7] Figures 2] shows comparisons of U-velocity results, where the velocity-slip effects are evident along the wall while some discrepancies are shown at the outlet boundary. Figure 3 shows comparisons of numerical and analytical temperature results. In general, the temperature fields have the same trends with large discrepancies. Figures 4-6 are based on four different cases: 1) no magnetic and electric fields; 2) $K=0$; 3) $K=0.5$; and 4) $K=0.9$. We intend to show some trends by comparing the results from these four cases. Nonuniform strength of sources terms are added to the X-momentum and the energy equations for the last two cases. Figure 4 shows the pressure distributions along the flow direction, with the linear pressure distributions subtracted out. The lines without soiled symbols are analytical results, while those with solid symbols represent the numerical simulation results for the last case. Though numerical solutions are obtained for all cases, here we merely present one case of $K=0.9$ for clarity. It is clear that the linear pressure gradient assumed in the Hartmann flow is not applicable here and it may result in inaccuracies if used carelessly. For these four test cases, at any specific station pressure level increases from case 1 to case 4, indicating that the extra magnetic field or electric field results in increasingly stronger impedance to the flow field. For Cases 1 and 2 , the numerical and analytical results are essentially the same; while for the last two cases, the nonlinearities become more appreciable. Larger discrepancies are found for cases 3 and 4 than those of cases 1 and 2. Figure 5 shows the different $\mathrm{U}$-velocity profiles for the four test cases at the middle station of the channel, $x / L=0.5$. The electric and magnetic fields are shown to strongly influence the velocity profiles. Numerical and analytical results are close. For clarity, only the numerical results of case 4 are shown. Figure 6 displays the profiles of velocity

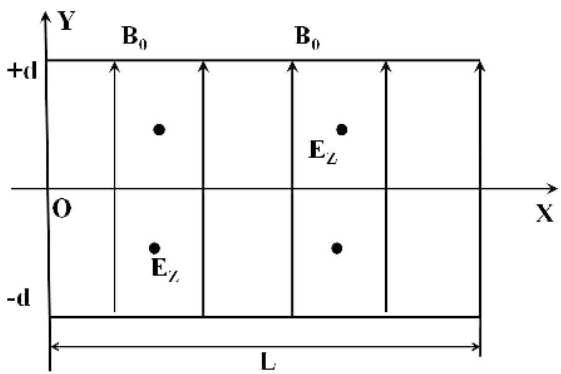

Fig. 1. Illustration of the problem, $B=\left(0, B_{0}, 0\right), E=\left(0,0, E_{z}\right)$

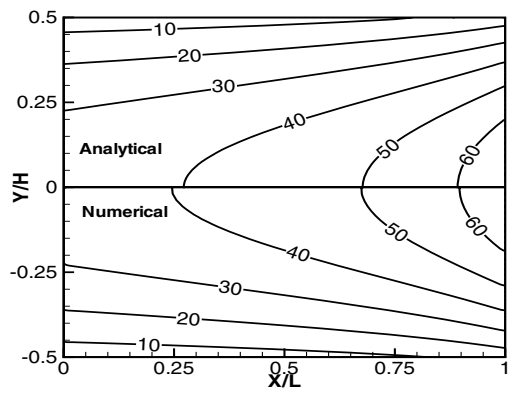

Fig. 2. Contours of U-velocity, $P=2$, $K=0.5, R_{b}=1 / \epsilon, R_{\sigma}=\epsilon, \epsilon=0.06$ 


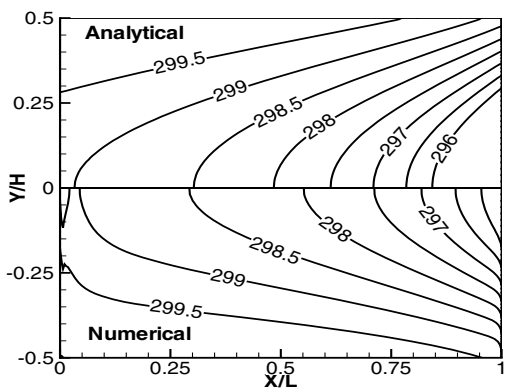

Fig. 3. Contours of temperature, $P=$ $2, K=0.5, R_{b}=1 / \epsilon, R_{\sigma}=\epsilon, \epsilon=0.06$

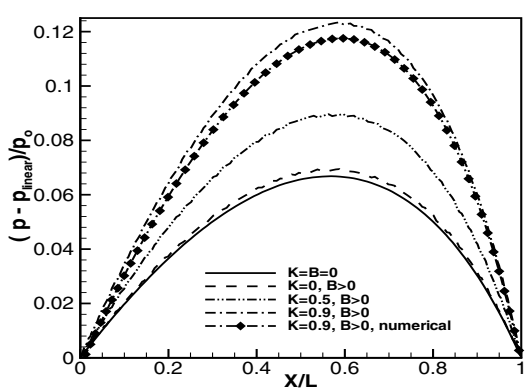

Fig. 4. Pressure distribution along the flow direction, $P=2, R_{b}=1 / \epsilon, R_{\sigma}=$ $\epsilon, \epsilon=0.06$

slips along the upper walls for these four test cases. In general, the slip velocities increase as gas flows downstream, and numerical results are found to agree with analytical results. Essentially, for the last two cases, the gas flows are both Fanno and Rayleigh flows due to the wall friction and Joule heating effects. Hence, with stronger Joule heating effects in case 4, the subsonic flow increases its speed more significantly towards the sonic speed than the that of case 3 . Now, we will examine the discrepancies found on the results. 1) The numerical simulation results are from the whole MGD equations, while the analytical solutions are based on the simplified asymptotic equations. If we consider the truncation error $O(\epsilon)$ as an upper limit, there is about $6 \%$ theoretical difference with $\epsilon=0.06 ; 2$ ) The analytical results are actually based on the assumption that the channel is in the middle of flow field without entrance effects; however, for numerical solutions, the entrance effects are not avoidable due to the presence of significant friction occurring at the inlet region. The uniform free stream needs to "adjust" to the channel flow around the inlet entrance. The inlet and outlet boundary condition treatment is very subtle, and the outlet boundary condition treatment is important since the gradients there are large. However, these end boundary effects are not included in the asymptotic solutions at all. Significant discrepancies therefore show up in the V-Velocity and temperature profiles. If we use further longer simulation case, most probably the results shall be better. 3) Betweens the analytical and numerical solutions, the pressure fields already have some discrepancies, and with the small length dimension, $d p / d x$ and $d p^{2} / d x^{2}$ must be huge quantities. For the temperature field, the $d p / d x$ term dominates in the coefficients, and $(d p / d x)^{2}$ appears in the coefficients $N_{5}$ and $N_{6}$. Hence, it is not surprising to see that the temperature profiles have poorer agreement than the velocity and pressure results. 4) Analytically, here we only consider the leading term for the electric and magnetic field effects but since they are coupled in the source terms for the momentum and energy equations, some nonlinear effects are possible. Figures 7 and 8 show the temperature profiles and the temperature gradients along the upper wall boundary. As discussed earlier, it is very 


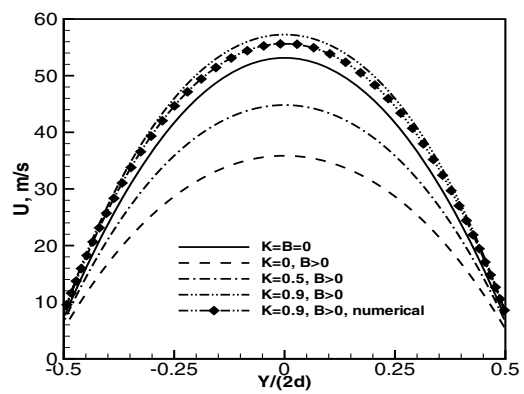

Fig. 5. Profiles of U-velocity distributions at the middle section, $x / L=0.5$, $P=2, R_{b}=1 / \epsilon, R_{\sigma}=\epsilon, \epsilon=0.06$

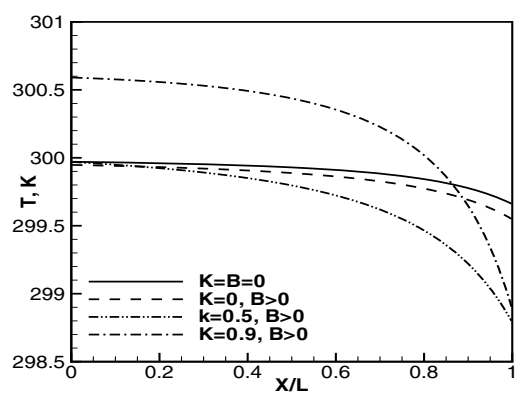

Fig. 7. Profiles of temperature distributions along the upper wall, $P=2$, $R_{b}=1 / \epsilon, R_{\sigma}=\epsilon, \epsilon=0.06$

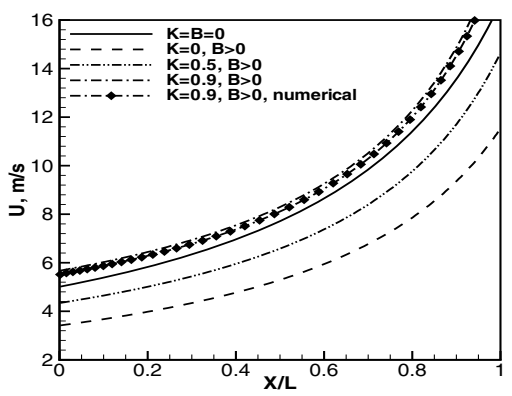

Fig. 6. Profiles of slip velocity along the wall, $y=d, P=2, R_{b}=1 / \epsilon$, $R_{\sigma}=\epsilon, \epsilon=0.06$

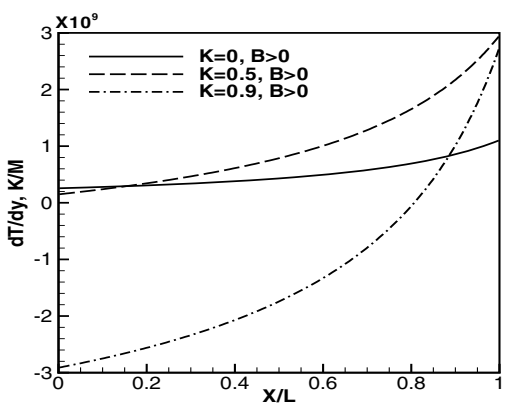

Fig. 8. Profiles of temperature gradient along the upper wall, $P=2, R_{b}=$ $1 / \epsilon, R_{\sigma}=\epsilon, \epsilon=0.06$

difficult to obtain accurate temperature profiles, especially when both electric and magnetic fields are considered. Hence, these plots only show the analytical results. For cases $K=0.5$ and $K=0.9$, there are Joule heating effects in the energy equation. For $K=0.9$, stronger Joule heating deposits more energy into the field, resulting in a much higher increase in the temperature field. As shown in Fig. 7. a portion of gas close to the wall is actually hotter than the wall. Correspondingly, for the last case, heat flux is transferred into the wall instead from the wall, as shown by Fig. 8. There, it also indicates one important effect associated with an MGD gas flow inside a microchannel: along the wall, even when the temperature change is very small, the normal temperature gradient can be huge because of the narrow channel height. 5. Hence, this shows that the heat transfer problem in microchannels is of practical importance. For Case 1 $(\mathrm{K}=0)$ without any electric and magnetic field effects, the temperature gradient reaches an order of $1 \times 10^{6} \mathrm{~K} / \mathrm{m}$. By contrast, with magnetic and electric field 
effects, $(K=0.9)$, the magnitude of temperature gradient becomes much larger and variant, as shown in Fig 8 .

\section{Conclusion}

We reported an analysis of rarefied MGD gas flows inside a 2D microchannel with velocity-slip and temperature-jump boundary conditions assuming that the magnetic Reynolds number is low and the flow is quasi-isothermal. By carefully comparing different orders of magnitude for the pressure drop, viscous shear stress at the channel wall, and the magnetic forces, two sets of parameters are selected and used to simplify the MGD equations. This study yields asymptotic solutions for velocity components, pressure and temperature. With stronger Joule heating effects, the Rayleigh process effects become significant, and the average pressure, velocity and temperature inside the channel increases. In general, the pressure gradient along the flow direction is nonlinear inside the channel, and the velocity and density distributions are nonuniform. Numerical solutions of the same formulation are obtained to validate these asymptotic solutions; explanations are provided for the discrepancies found between these solutions.

Future work may include other asymptotic solutions with different set of parameters, and the corresponding solutions to three-dimensional or axially symmetric flows.

\section{References}

1. Arkilic, E.B., Schmidt, M.A., Breuer, K.S.: Gaseous Slip Flow in Long Microchannels. J. Microelectromech. Syst. 6(2) (1997)

2. Zohar, Y., Lee, S.Y.K., Lee, W.Y., Jiang, L., Tong, P.: Subsonic Gas Flow in a Straight and Uniform Microchannel. J. Fluid Mech. 472 (2002)

3. Cai, C., Boyd, I., Fan, J., Candler, G.V.: Direct Simulation Methods for Low-Speed Microchannel Flows. J. Thermophys Heat Trans. 14 (2000)

4. Xu, K., Li, Z.: Microchannel Flow in the Slip Regime: Gas-Kinetic BGK-Burnett Solutions. J. Fluid Mech. 513 (2004)

5. Cai, C., Sun, Q., Boyd, I.D.: Gas Flows in Microchannels and Microtubes. J. Fluid Mech. 589 (2007)

6. Boyd, T.J.M., Sanderson, J.J.: Plasmadynamics, Nelson, London (1969)

7. Gaitonde, D.V., Poggie, J.: Simulation of MHD Flow Control Techniques. AIAA paper 2000-2326

8. Agarwal, R.K.: Lattice Boltzmann Simulations of Magnetohydrodynamics Slip Flow in Microchannels. AIAA paper 2005-4782

9. Naterer, G.F., Camberos, J.A.: Entropy Based Design of Thermofluid and Microfluidc Systems. CRL Pr I Llc (June 2007)

10. Cai, C., Boyd, I.D.: Compressible Gas Flows in a Two-Dimensional Planar Microchannel. J. Thermophys Heat Transs. 21(3), 608-615 (2007) 